\title{
La relación de consumo en el Derecho argentino
}

\section{The Consumer Relationship in Argentine

Carlos Eduardo Tambussi* https://orcid.org/0000-0003-0444-7937

http://dx.doi.org/10.21503/lex.v19i27.2255

* Carlos Eduardo Tambussi, Abogado, Universidad de Buenos Aires (1991). Secretario del Juzgado Nro. 18 Secretaria 35 del Fuero Contencioso Administrativo y Tributario de la CABA (2013-actualidad). Integrante de la Comisión para la redacción del Código Procesal de la Justicia en las Relaciones de Consumo de la CABA (Resolución 423/16 de la Subsecretaria de Justicia CABA) y de la Comisión para la Reforma de la Ley 24240 (Programa Justicia 2020). Co Director del Programa de Actualización en Derecho del Consumo (Convenio AABA-UBA). Profesor Adjunto Regular: Universidad de Buenos Aires, Facultad de Derecho, Cátedra: Dr. Roberto Saba, en las asignaturas Derechos Humanos y Garantías y Protección Constitucional de Consumidores y Usuarios. Argentina.

Correo electrónico: cetambu@uolsinectis.com.ar

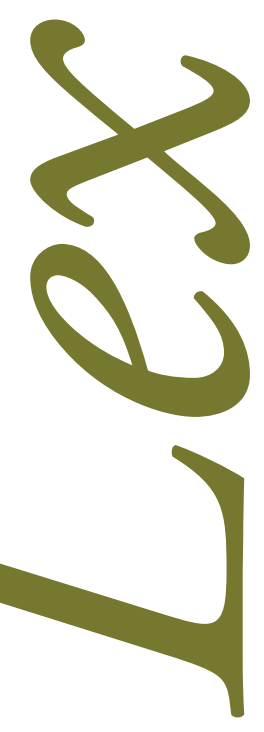




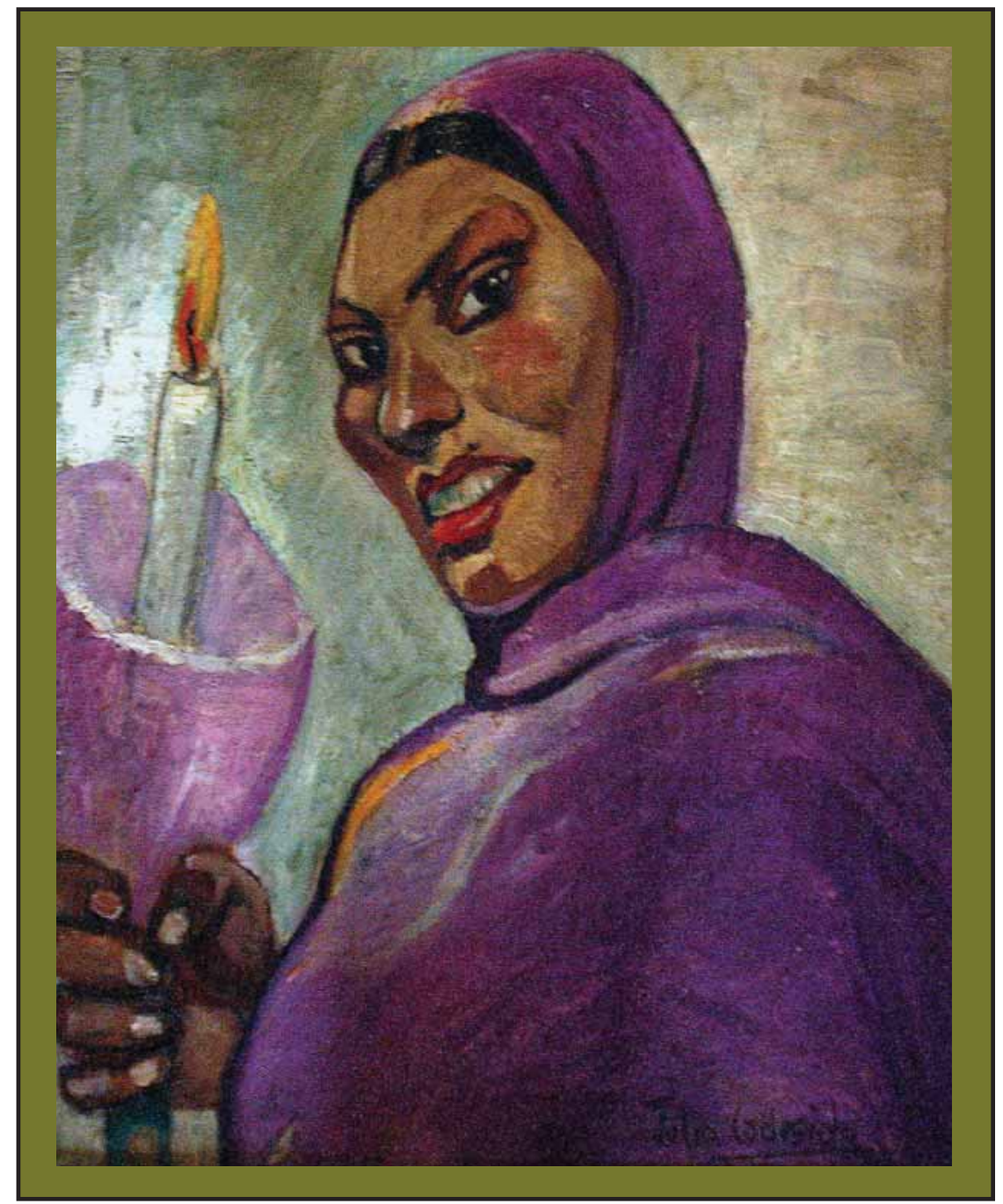

Morena. Colección Néstor Benavides. Julia Codesido pintora peruana del indigenismo (Lima 1883-1979). 


\section{RESUMEN}

La relación de consumo es el objeto de estudio del derecho del consumidor. En el caso argentino, constituye una noción de origen constitucional que es punto de partida de la disciplina y que a la par del desarrollo legislativo de las normas sobre la materia, fue interpretada con un alcance significativo y amplio, comprensivo de todas las situaciones en que el consumidor se ve involucrado y de los riesgos que lo acechan. La verificación de su existencia en un caso provoca que tanto en la interpretación de las normas de fondo como en lo instrumental de las garantías se apliquen los principios protectorios del derecho del consumidor.

Palabras clave: Consumidores y usuarios, relación de Consumo, Constitución Nacional Argentina. Código Civil y Comercial, Ley Argentina de Defensa del Consumidor.

\section{ABSTRACT}

The consumer relationship is the object of study of consumer law. In the Argentine case, it constitutes a notion of constitutional origin that is the starting point of the discipline and that, along with the legislative development of the norms on the matter, was interpreted with a significant and broad scope, comprehensive of all the situations in which the consumer is involved and in the risks that threaten him. The verification of its existence in a case, causes that both in the interpretation of the substantive rules and in the instrumentalities of the guarantees, the protective principles of consumer rights are applied.

Key words: Consumers and users, consumption ratio, Argentine National Constitution, Civil and Commercial Code, Argentine Law for the Defense of the Consumer. 


\section{INTRODUCCIÓN, UN CONCEPTO CONSTITUCIONAL CON RECEPCIÓN LEGISLATIVA}

Con anterioridad a la reforma constitucional de 1994, se consideraba a los derechos del consumidor como incluidos dentro de los implícitos del Artículo 33 de la Carta Magna, como relacionados con la salud, la dignidad de la persona, a más del reconocimiento expreso del derecho de propiedad ${ }^{1}$.

El constituyente de 1994 los incorporó, llevándolos a su más alta consideración en consonancia con la corriente que se venía dando dentro del constitucionalismo provincial ${ }^{2}$ y en el derecho extranjero ${ }^{3}$, consagrándolos expresamente en el artículo 42 de su texto:

Artículo 42 - Los consumidores y usuarios de bienes y servicios tienen derecho, en la relación de consumo, a la protección de su salud, seguridad e intereses económicos; a una información adecuada y veraz; a la libertad de elección, y a condiciones de trato equitativo y digno.

Las autoridades proveerán a la protección de esos derechos, a la educación para el consumo, a la defensa de la competencia contra toda forma de distorsión de los mercados, al control de los monopolios naturales y legales, al de la calidad y eficiencia de los servicios públicos, y a la constitución de asociaciones de consumidores y de usuarios.

La legislación establecerá procedimientos eficaces para la prevención y solución de conflictos, y los marcos regulatorios de los servicios públicos de competencia nacional, previendo la necesaria participación de las asociaciones de consumidores y usuarios y de las provincias interesadas, en los organismos de control.

1. Nota del autor: sin perjuicio de lo expresado en este párrafo, la ausencia de legislación específica en materia de consumo dejaba la aplicación de una visión realista de este fenómeno al infrecuente protagonismo judicial. No siendo común por entonces ese rol, se daban decisorios como el del caso de la Cámara Nacional de Apelaciones en lo Comercial, Sala D, 29/05/1992, "Befumo, Juan E. v. Sevel S.A. y otro", publicado en JA 1993-IV-331, cuya lectura merece hacerse.

2. Nota del autor: a partir de 1983 muchas constituciones provinciales fueron reformadas integralmente en la República Argentina.

3. Nota del autor: las fuentes extranjeras de la ley argentina de defensa del consumidor son la Ley General Española de 1984, la ley federal Mexicana de 1975, y los antecedentes legislativos de Canadá (1982), Francia (1973), Venezuela (1974) y Brasil (Código de Defensa del Consumidor, ley 8708/90) y a nivel internacional las Directrices de las Naciones Unidas para la Protección del Consumidor 
Queda expresamente plasmada la trascendencia que ha tenido para el constituyente y para la ciencia jurídica la elevación a rango constitucional de estos derechos con su correlato de beneficios y garantías para la población en general en su vida de todos los días. A su vez, ha producido un impacto profundo en el régimen contractual y extracontractual, modificando el mundo del derecho privado, coexistiendo con el mismo pero con una naturaleza, ámbito de aplicación y alcances especiales, bifurcando las relaciones jurídicas en aquellas que son de consumo (con un perfil propio y distintivo), y las que no lo son. Por ende, ha dividido el universo jurídico ${ }^{4}$.

Del texto de la norma constitucional surge literalmente la expresión "relación de consumo" como ámbito de aplicación del derecho de consumidores y usuarios, concepto que no aparecía mencionado en el estatuto especial de carácter legislativo, la ley $24240^{5}$ de Defensa del Consumidor que había construido su enfoque protectorio exclusivamente en torno de la idea de consumidor como contratante, en su texto original de 1993.

Tuvieron que transcurrir casi quince años de su vigencia para que el precepto se incorporara mediante la ley 26361 del 12 de marzo de 2008, promulgada parcialmente el día 3 de abril de 2008 y publicada en el Boletín Oficial de la Nación en fecha 7 de Abril de 2008. No podría completarse el sentido de la reforma en este punto, sin agregar al texto legal un concepto de relación de consumo, el cual se contempla en el primer párrafo del artículo tercero y de esa manera hacer honor a la redacción del artículo 42 de la Constitución Nacional, quedando plasmado en el artículo 3 de la norma especial:

Artículo $3^{\circ}$ - Relación de consumo. Integración normativa. Preeminencia.

Relación de consumo es el vínculo jurídico entre el proveedor y el consumidor o usuario.

Las disposiciones de esta ley se integran con las normas generales y especiales aplicables a las relaciones de consumo, en particular la Ley No 25.156 de Defensa de la Competencia y la Ley No 22.802 de Lealtad Comercial o las que en el futuro las reemplacen. En caso de duda sobre la interpretación de los principios que establece esta ley prevalecerá la más favorable al consumidor.

Las relaciones de consumo se rigen por el régimen establecido en esta ley y sus reglamentaciones sin perjuicio de que el proveedor, por la actividad que desarrolle, esté alcanzado asimismo por otra normativa especifica.

4. Un auténtico "big bang" de nuestro ordenamiento jurídico, de manera tal que estamos frente a un cambio de "paradigma" (Francisco Junyent Bas - Candelaria del Cerro, "Aspectos procesales en La Ley de defensa del consumidor", en La Ley, (14/06/2010):1.

5. Nota del autor: la norma fue sancionada por el Congreso el 22 de septiembre de 1993 y fue parcialmente promulgada por el Presidente de la Nación, con fecha 13 de octubre de 1993, mediante el decreto 2089/93. 
El legislador incorporó la noción de relación de consumo como el "vinculo jurídico entre el proveedor y el consumidor o usuario". Esta formulación fue criticada ya que el término "vínculo" parece aludir principalmente a un carácter contractual, lo cual entra en contradicción con la incorporación al texto legislativo -dentro de los considerados consumidores- a quien no contrató a título oneroso y a quien no tiene vinculación contractual con el proveedor (artículos 1 y 2 de la ley 24240 hoy vigente), agregándose además que también se consideraba consumidor a quien "de cualquier manera esté expuesto a una relación de consumo" (párrafo final, hoy derogado, del art. 1), pero sin serlo.

El artículo se completa con la mención a que las disposiciones de la ley se integran con las normas generales y especiales aplicables a las relaciones de consumo, en particular las leyes de Defensa de la Competencia y de Lealtad Comercial, estándose siempre en materia interpretativa por la aplicación de la norma más favorable al consumidor.

Vigente desde el año 2015, el Código Civil y Comercial de la Nación (ley 26994), replicó el concepto de relación de consumo de la ley 24240 y eliminó de la definición o concepto de consumidor al llamado "expuesto", también conocido como by stander al que hiciéramos referencia.

Código Civil y Comercial de la Nación. Art. 1092. — Relación de consumo. Consumidor.

Relación de consumo es el vínculo jurídico entre un proveedor y un consumidor. Se considera consumidor a la persona humana o juridica que adquiere o utiliza, en forma gratuita u onerosa, bienes o servicios como destinatario final, en beneficio propio o de su grupo familiar o social.

Queda equiparado al consumidor quien, sin ser parte de una relación de consumo como consecuencia o en ocasión de ella, adquiere o utiliza bienes o servicios, en forma gratuita u onerosa, como destinatario final, en beneficio propio o de su grupo familiar o social.

Alguna doctrina entiende que la relación de consumo debió haber sido incluida en el Título I, "Obligaciones en General" en lugar del referido a los contratos, ya que siendo la relación de consumo el vínculo jurídico entre consumidor y proveedor, muchas veces puede resultar de un acto unilateral, o de un hecho, resultando inapropiado que el concepto de relación de consumo sea el "portal de ingreso" al tratamiento del título de los contratos de consumo ${ }^{6}$.

En el articulado del Código, aparecen contratos caracterizados como comprensivos de una relación de consumo:

- Artículo 2100.- Relación de consumo. La relación entre el propietario, emprendedor, comercializador y administrador del tiempo compartido con quien adquiere o utiliza el derecho de uso periódico se rige por las normas que regulan la relación de consumo, previstas en este Código y en las leyes especiales.

6. Nota del autor: Esteban Arias Cau, Federico Álvarez Larrondo y Dante Rusconi, entre otros. 
- Artículo 2111.- Relación de consumo. La relación entre el propietario y el administrador del cementerio privado con los titulares de las parcelas se rige por las normas que regulan la relación de consumo previstas en este Código y en las leyes especiales.

De esta manera, al incorporarse el régimen tuitivo consumidor al Código Civil y Comercial y convivir con el estatuto especial ley 24240, el legislador mantiene el concepto base de la protección constitucional y legal de estos derechos: la relación de consumo, mediante la formulación de una definición normativa, que aun dentro de las criticas señaladas, entendemos en la amplitud que se venía gestando y aplicando en su evolución a mayores niveles protectorios (excepto en lo referente a la exclusión del consumidor expuesto), abarcando "todas las situaciones en que el sujeto es protegido: antes, durante y después de contratar; cuando es dañado por un ilícito extracontractual, o cuando es sometido a una práctica del mercado; cuando actúa individualmente o cuando lo hace colectivamente.

\section{ALCANCE DE LA RELACIÓN DE CONSUMO}

Siendo la existencia de una relación de consumo el presupuesto el elemento que decide el ámbito de aplicación del derecho del consumidor debe comprender todas las situaciones posibles" ". La relación de consumo contempla, en suma, todos los aspectos en los que se manifiesta y verifica la vulnerabilidad del consumidor, y la necesidad de su protección.

La protección se extiende a situaciones extracontractuales, ya que lo protegido no es el hecho de contratar sino de consumir ${ }^{8}$. Por lo que la relación de consumo es comprensiva del contrato en toda su extensión (etapa precontractual, etapa poscontractual, sucesores singulares, beneficiarios de estipulaciones a favor de terceros, etc.), actos unilaterales de los proveedores, vínculos no contractuales de derecho público y privado, y hechos jurídicos ${ }^{9}$. La norma, en la interpretación que viene teniendo en la ley 24240 "va mucho más allá, porque ni siquiera se requiere probar la intención de contratar en los casos en que el sujeto protegido es un consumidor expuesto a prácticas comerciales, o un usuario" 10 .

La extensión del concepto cobra importante significación en material reparatoria, "ya que el consumidor en sentido amplio tiene derecho al ámbito tuitivo del Derecho del Consumidor y contará con las herramientas preventivas y reparatorias previstas por dicho régimen", que comprende principios protectorios, normas de orden público, y criterios procesales conducentes a facilitar el acceso a la justicia.

7. Ricardo Luis Lorenzetti, Consumidores, (Santa Fe: Rubinzal-Culzoni, 2 ${ }^{\mathrm{a}}$ ed 2009), 74.

8. "Conforme el Art. 42 de la Constitución Nacional, que se refiere a los derechos de los consumidores y usuarios en la relación de consumo, que es un concepto más amplio que el de contrato para consumo, la distinción entre responsabilidad contractual o extracontractual no debe ser aplicada para resolver cuestiones derivadas de dicha relación" (Juzgado de Primera Instancia en lo Civil, Comercial y Minas Nro. 13 de Mendoza, 21/1272009," Maldonado, Juan Carlos c. Faingold, Marcos Blas y otros", LLGran Cuyo 2010 -abril-), p.292).

9. Sergio Barocelli y Esteban Arias Cau, "Necesaria acreditación de una relación de consumo para los daños punitivos", La Ley, (05/09/2014): 4.

10. Ricardo Luis Lorenzetti, Consumidores, op. cit., 127. 
La relación de consumo también se entabla cuando se trata de cosas usadas ${ }^{11}$ ya sean comercializadas profesional u ocasionalmente, con lo que en este punto se verifica la amplitud de las relaciones comprendidas en la norma.

Por esa razón, ha sido definida como "el vínculo jurídico de fuente legal que liga al proveedor de bienes o servicios con el consumidor que los adquiere o utiliza como destinatario final, así como con todos aquellos que se ven afectados por sus consecuencias o, en general, por la actividad de los proveedores"12.

De esta manera, "los distintos vínculos contractuales anudados a lo largo de la cadena de producción son considerados todos ellos como una unidad ante la cual se consagran los derechos de los consumidores. Ya no se persigue la protección del sujeto ante cada uno de los contratos que componen la cadena o sistema, sino por el contrario, la tutela del mismo frente a la realidad negocial compleja"13.

Esa complejidad está dada:

- En que desde la producción misma del bien o servicio hay consecuencias que pueden repercutir en el consumidor, que generan la responsabilidad de los integrantes de la "cadena de valor" ${ }^{\text {", }}$, aunque el consumidor sólo trate con el comercializador final.

- En que la relación de consumo es un concepto más amplio que el de contrato para consumo, y por ende la distinción entre responsabilidad contractual o extracontractual no debe ser aplicada para resolver cuestiones derivadas de dicha relación. A su vez, impone al proveedor la protección del consumidor más allá del producto o servicio adquirido, incluyendo el "iter" completo en el cual se desenvuelve la vinculación de los sujetos, sea antes, durante o después de la eventual contratación. Así, dentro de la relación de consumo, no solamente queda incluido como fuente posible el contrato, sino también actos jurídicos unilaterales (publicidad, meras tratativas, ofertas a personas determinadas o indeterminadas), los hechos ilícitos, o los hechos lícitos como las prácticas comerciales (actos del oferente encaminados

11. El fabricante es responsable por los desperfectos que presentó un vehículo usado, adquirido en una concesionaria, toda vez que su responsabilidad objetiva surge del Art. 40 de la ley 24240 ante el daño provocado al consumidor, resultante del vicio de la cosa, del cual únicamente se hubiera liberado total o parcialmente demostrando que la causa del daño se debe a culpa inexcusable de la víctima o al hecho de un tercero por quien no deba responder. (Cámara Nacional de Apelaciones en lo Comercial, sala D, 30/03/2011, "Di Marco Rómulo Dellmo c. PSA Peugeot Citroën Argentina S.A", RCyS 2011-VIII, p.233).

12. Diego H. Zentner, Contrato de consumo, (Buenos Aires: La Ley, 1a edición, 2010), 68.

13. Federico Manuel Álvarez Larrondo, “Consumidores: 20 años que cambiaron el Derecho argentino”, $L a$ Ley Sup. Const. (octubre, 2014): 7.

14. "La responsabilidad del vendedor directo frente al adquirente de una computadora es contractual y tiene como fundamento una obligación de seguridad o garantía, que asume el enajenante y acompaña el contrato oneroso, por lo cual más allá de una eventual acción de repetición contra aquel que se entienda el real culpable del daño, corresponde que todos los que integran la cadena de producción y comercialización respondan por el hecho dañoso ocasionado por el producto o por el incumplimiento de la garantía otorgada" (Cámara Nacional de Apelaciones en lo Comercial, sala D, 04/08/2010, “Madera, Graciela María c. Omnistar de Super Imagen S.R.L., RCyS 2011-II , p. 213). 
a la captación del cliente, aunque no signifiquen oferta o una actividad destinada a contratar con un sujeto determinado), incluyendo la publicidad en general, las promociones, el marketing) ${ }^{15}$.

De modo que la relación de consumo se integra entre aquel que asume un deber jurídico (proveedor) frente al que ostenta un derecho subjetivo (consumidor), con prescindencia de la fuente, debiéndose poner el foco en la razón por la que se vinculan (no siempre precisas), y las consecuencias jurídicas de ese vínculo ${ }^{16}$.

En coincidencia y por nuestra parte, creemos que hubiera sido más preciso definirla como el "vínculo con consecuencias jurídicas" entre consumidor y proveedor, a fin de aventar posibles residuales interpretaciones que asocien la voz "vínculo jurídico" solamente con el contrato, y para comprender la diversidad de fuentes antes señaladas, en especial las que se basan en hechos.

En la actualidad, la normativa de consumo se encuentra en etapa de discusión en la Argentina, encontrándose en estado parlamentario iniciativas de reforma al estatuto especial, y que propugnan la sanción de un verdadero código de defensa del consumidor ${ }^{17}$.

La propuesta de reforma recoge la amplitud del concepto de relación de consumo, determinando en el artículo primero de la norma (que mantiene la formulación del mismo) como fuentes de la relación de consumo "a un hecho o acto jurídico, unilateral o bilateral, una práctica o una técnica de marketing". Y a su vez establece que puede resultar de la "tipificación legal, de la voluntad individual o inferirse a través de la interpretación judicial”.

Se verifica el primer caso, cuando la misma norma define como relación de consumo una actividad (v.gr. el artículo 2100 del Código Civil y Comercial transcripto supra). Para el segundo ejemplo habrá que analizar la correspondencia en el caso del presupuesto de los conceptos legislativos de consumidor y proveedor, y en el tercer supuesto jugará la interpretación judicial de casos dudosos conforme al principio de primacía de la realidad.

De esta manera, conforme sus fundamentos expresados en la elevación al tratamiento legislativo se proponen para construir todo su esquema protectorio "de la noción de relación de consumo, a la que se tipifica de modo amplio a partir de la enunciación de sus fuentes”. Se recoge así, la resignificación de la amplitud del concepto, abarcativo de un amplio universo de situaciones en las que el consumidor está necesitado de protección ${ }^{18}$.

15. Claudio A. Castagnet, “Contratos de Consumo”, DJ (25/01/2006): 147.

16. Rinessi Juan Antonio, Relación de consumo y derechos del consumidor, (Buenos Aires: Astrea, 2006), 3.

17. Nota del autor: proyecto presentado en diciembre de 2018, el marco del Programa Justicia 2020 en trámite parlamentario por ante la Cámara de Diputados de la Nación y resultado del trabajo de una comisión formada por abogados de la matrícula, académicos, autoridad de aplicación, magistrados, provenientes de distintas provincias. La integran Gabriel Stiglitz, Fernando Blanco Muiño, Carlos Hernández, Belén Japaze, Sebastián Picasso, Leonardo Lepiscopo, Gonzalo Sozzo, Federico Ossola, Roberto Vázquez Ferreyra, María Eugenia D’Archivio, Javier Wajntraub y el autor de este trabajo. 18. Laura Chiesa y Bibiana Rodríguez, "La publicidad en la relación de consumo y el rol del Estado" en elDial.com, Cita DC18DE. 


\section{TRATAMIENTO JURISPRUDENCIAL}

La jurisprudencia recepta la relación de consumo como presupuesto de aplicación del régimen tuitivo:

"El vínculo jurídico existente entre un proveedor de créditos y un consumidor o usuario que solicitó un mutuo para realizar una reforma en su hogar se subsume dentro de una relación de consumo, pues, si bien el monto del contrato era elevado para considerarlo un crédito personal, no resulta incongruente con el destino aludido, máxime cuando es carga del mutuante demostrar el destino de los fondos (Art. 902, Código Civil)." ${ }^{19}$.

"La relación de consumo se anuda mediante el mero contacto social entre el proveedor y el consumidor o usuario, en los términos que fija la propia ley 24240, y no resulta necesario que exista o subsista un vínculo contractual”20.

"El servicio de subterráneo no solo reporta un servicio público sino que también se encuentra regido por las normas de consumo, toda vez que se ofrece un servicio en los términos del Art. 2 de la ley 24240 y el pasajero se constituye en usuario a la luz del Art. 1 de la citada normativa, configurando así una relación de consumo - Art. 3, ley de defensa del consumidor- ${ }^{21}$.

“Conforme el artículo 42 de la Constitución Nacional, que se refiere a los derechos de los consumidores y usuarios en la relación de consumo, que es un concepto más amplio que el de contrato para consumo, la distinción entre responsabilidad contractual o extracontractual no debe ser aplicada para resolver cuestiones derivadas de dicha relación"22.

"La relación de consumo definida por el artículo $3^{\circ}$ de la ley 24.240, impone al proveedor la protección del consumidor más allá del producto o servicio adquirido, incluyendo el íntegro trayecto en el cual se desenvuelve la vinculación de los sujetos, sea antes, durante o después de la eventual contratación"23.

19. Cámara de Apelaciones en lo Civil y Comercial de Necochea, 18/07/2014, "F. S. A. c. S., J. F. y otros s/ cobro ejecutivo", LLBA, 2014 (noviembre), 1149. La Ley On Line AR/JUR/45734/2014.

20. Cámara Nacional de Apelaciones en lo Civil, sala A, 27/12/2012, "Waibsander, Eduardo Basilio c. Metrovías S.A. s/ daños y perjuicios” En La Ley On Line. Cita: AR/JUR/78063/2012.

21. Cámara de Apelaciones en lo Contencioso administrativo y Tributario de la Ciudad Autónoma de Buenos Aires, sala I, 14/11/2014, "Metrovias S.A. (resol. 103) c. Ente Único Regulador de Servicios Públicos de la Ciudad de Buenos Aires s/ otros rec. judiciales contra res. pers. públicas no est.", La Ley Online, AR/JUR/79191/2014.

22. Juzgado de 1a Instancia en lo Civil, Comercial y Minas Nro. 13 de Mendoza, 21/12/2009, "Maldonado, Juan Carlos c. Faingold, Marcos Blas y otros", LL Gran Cuyo (abril,2010): 292.

23. Cámara 4a de Apelaciones en lo Civil, Comercial, Minas, de Paz y Tributaria de Mendoza, 04/03/2009, “González, Yolanda c. Supermercados Norte S.A", LL Gran Cuyo (junio, 2009): 500. 


\section{CONCLUSIONES}

Habrá relación de consumo siempre que existan un consumidor y un proveedor en los términos de sus alcances y definiciones legislativas, por una multiplicidad de causas o fuentes que construyan un vinculo con consecuencias jurídicas, sin importar su origen contractual o extracontractual, aplicando el principio de primacía de la realidad en el marco de las complejas relaciones y procesos que interrelacionan a estos actores, en un determinado contexto.

En suma, el concepto sub-análisis importa:

- La conjunción de la protección constitucional del Art. 42 con la esfera de protección legal, ya consagrada en la definición de relación de consumo contenida en la ley 24240 a partir del año 2008 y ratificada por el Código Civil y Comercial desde el año 2015.

- Un concepto de cobertura de protección que conlleva la necesidad de conjugar el criterio de aplicación del régimen tuitivo consumidor solamente ante la acreditación de la existencia de una relación de consumo, cuya mutua presencia es condición para la verificación de la relación, atento su propia formulación como vínculo intersubjetivo (existirá cuando estemos en presencia de un consumidor en términos del Art. 1 y un proveedor en el sentido y alcance del Art. 2, en ambos casos de la ley 24240, y en función de los conceptos que aporta el nuevo código, aplicándose en la casuística la interpretación más amplia en el sentido de inclusión en el sistema protectorio). La relación de consumo es, como se ha dicho, la llave del sistema ${ }^{24}$ y el ámbito de aplicación del derecho del consumidor, siendo su punto de partida.

- Lo antes señalado importa que va a comprender toda circunstancia "que rodea, se refiere o constituya un antecedente o sea consecuencia de la actividad encaminada para satisfacer la demanda de bienes y servicios para destino final de consumidores y usuarios" 25 , incluyendo entre ellas el simple riesgo, atento la naturaleza preventiva del derecho de daños.

- Cuando en un caso estamos en presencia de una relación de consumo, se aplica el derecho de consumidor y sus principios. En materia de garantías, esto importa:

1) La posibilidad de echar mano a elementos procesales equilibrantes en la acción de consumo: aplicación del procedimiento más abreviado, goce del beneficio de justicia gratuita, y admisión de la teoría de las cargas dinámicas de la prueba.

2) La aplicación de aspectos propios de la acción de consumo, originados en el derecho de fondo, con influencia procesal: aplicación de los principios protectorios del régimen tuitivo consumidor, el carácter de orden público de las normas y la operatividad de las mismas.

24. Esteban Arias Cau - Sergio Barocelli, "Necesaria acreditación de una relación de consumo para los daños punitivos", La Ley (05/09/2014): p.4.

25. Juan Farina "Relación de consumo" en JA, 1995-I-855 
3) La aplicación de aspectos distintivos del procedimiento de consumo relacionados con la reparación de daños: responsabilidad objetiva y solidaria, vigencia del principio de reparación integral y posibilidad de solicitar la aplicación de daño punitivo.

\section{REFERENCIAS}

- Álvarez Larrondo Federico Manuel, "Consumidores: 20 años que cambiaron el Derecho argentino”, Argentina: La Ley, Suplemento Constitucional, (octubre 2014).

- Barocelli Sergio y Arias Cau Esteban Javier, "Necesaria acreditación de una relación de consumo para los daños punitivos", Argentina: La Ley, (05/09/2014).

- Cámara Nacional de Apelaciones en lo Comercial, sala D, 30/03/2011, "Di Marco Rómulo Dellmo c. PSA Peugeot Citroën Argentina S.A”, RCyS 2011-VIII, p. 233.

- Cámara Nacional de Apelaciones en lo Comercial, sala D, 04/08/2010,”Madera, Graciela María c. Omnistar de Super Imagen S.R.L.", RCyS 2011-II , p. 213.

- Cámara de Apelaciones en lo Civil y Comercial de Necochea, 18/07/2014, "F. S. A. c. S., J. F. y otros s/ cobro ejecutivo", LLBA 2014 (noviembre), 1149, La Ley, On Line AR/JUR/45734/2014.

- Cámara Nacional de Apelaciones en lo Civil, sala A, 27/12/2012, "Waibsander, Eduardo Basilio c. Metrovías S.A. s/daños y perjuicios”. En La Ley On Line. Cita: AR/JUR/78063/2012.

- Cámara de Apelaciones en lo Contencioso administrativo y Tributario de la Ciudad Autónoma de Buenos Aires, sala I, 14/11/2014, "Metrovias S.A. (resol. 103) c. Ente Único Regulador de Servicios Públicos de la Ciudad de Buenos Aires s/ otros rec. judiciales contra res. pers. públicas no est.", La Ley Online, AR/JUR/79191/2014.

- Cámara 4a de Apelaciones en lo Civil, Comercial, Minas, de Paz y Tributaria de Mendoza, 04/03/2009, “González, Yolanda c. Supermercados Norte S.A”, LL Gran Cuyo (junio, 2009), p.500.

- Castagnet, Claudio “Contratos de Consumo”, Doctrina Judicial J 25/01/2006.

- Chiesa, Laura y Rodríguez Bibiana "La publicidad en la relación de consumo y el rol del Estado" en EL Dial.com

- Farina Juan "Relación de consumo” en Jurisprudencia Argentina, 1995-I.

- Junyent Bas Francisco y Del Cerro, Candelaria, “Aspectos procesales en La Ley de defensa del consumidor", en La Ley, (14/06/2010). 
- Juzgado de Primera Instancia en lo Civil, Comercial y Minas Nro. 13 de Mendoza, 21/127/2009, "Maldonado, Juan Carlos c. Faingold, Marcos Blas y otros", La Ley Gran Cuyo (abril 2010), p. 292.

- Lorenzetti, Ricardo Luis, Consumidores. Argentina: Santa Fe Rubinzal-Culzoni,, 2a ed San, 2009.

- Rinessi Juan Antonio. Relación de consumo y derechos del consumidor. Buenos Aires, Astrea, 2006.

- Zentner, Diego. Contrato de consumo, Buenos Aires: La Ley, 2010.

RECIBIDO: $12 / 02 / 2021$

APROBADO: 30/05/2021 


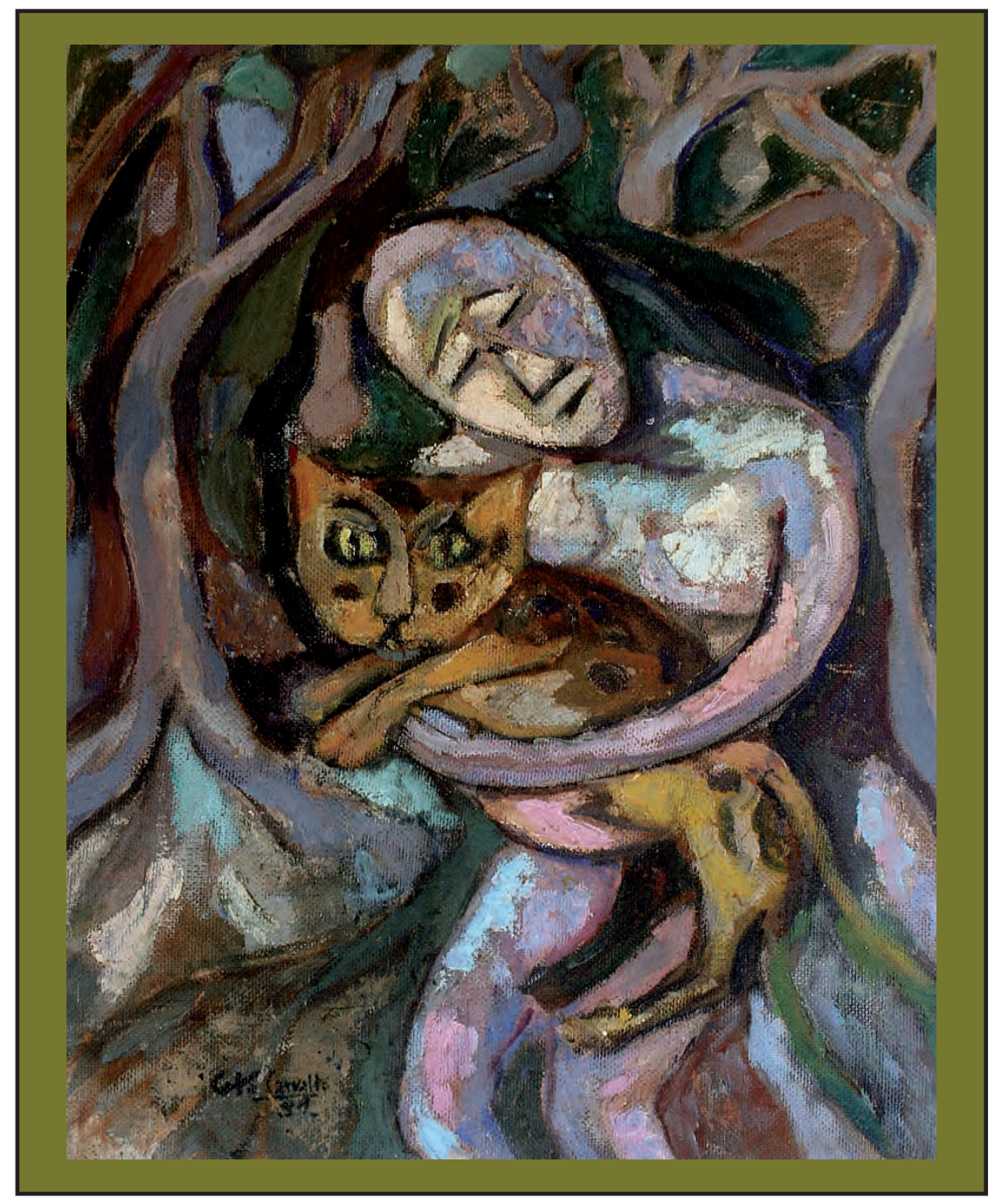

Mujer y tigre. Carlota Carvallo pintora peruana del indigenismo (Lima 1909-1980). 